\title{
Thymus Dysfunction in the Development of Type 1 Diabetes and Endocrine Autoimmune Diseases
}

\author{
Vincent Geenen ${ }^{1}$ and Olivier Dardenne ${ }^{2}$
}

1. Director of Research; 2. Research Associate, Centre of Immunoendocrinology, Institute of Pathology, University of Liège

DOI:10.17925/EE.2009.05.00.24

\begin{abstract}
The discovery that thymic epithelium from many species expresses a large repertoire of genes encoding neuroendocrine and other tissuerestricted antigens has radically changed our knowledge of the pathogenic mechanisms underlying the development of organ-specific autoimmune diseases such as type 1 diabetes and autoimmune endocrine diseases. Rather than a breakdown of immunological selftolerance in periphery, there is mounting evidence that the diabetogenic autoimmune response may first arise from a thymus dysfunction in the central programming of $\beta$-cell self-tolerance. Insulin-like growth factor 2 (IGF-2) is the dominant member of the insulin gene/protein family expressed in thymic epithelial cells (TECS) from different species, and Igf2 $\%$ mice fail to programme complete tolerance to insulin. Based on the homology between insulin, the primary and immunogenic auto-antigen of type 1 diabetes, and IGF-2, the tolerogenic selfantigen of the insulin family, the design of a regulatory/negative self-vaccination for prevention against type 1 diabetes has been proposed and is under development.
\end{abstract}

\section{Keywords}

Thymus, type 1 diabetes, self-tolerance, insulin-like growth factor 2, insulin

Disclosure: These studies were supported by the Fund of Scientific Medical Research of Belgium (convention 3.4508.04), the Fonds Leon Fredericq (Liège University Hospital), the Walloon Region (Waleo 2 convention Tolediab), the European Association for the Study of Diabetes (EASD, Düsseldorf, Germany) and the EU FP6 Integrated Project EuroThymaide (contract LSHB-CT-2003-503410/www.eurothymaide.org).

Received: 6 May 2009 Accepted: 17 July 2009

Correspondence: Vincent Geenen, Centre of Immunoendocrinology, Institute of Pathology CHU-B23, B-4000 Liège-Sart Tilman, Belgium. E: vgeenen@ulg.ac.be

In distant species and invertebrates, the foundations of the neuroendocrine and immune systems have co-existed until now without any apparent problem. Some 470 millions years ago, while gene conversion already determined some rudiments of immune diversity in primitive vertebrates (such as jawless fishes), adaptive immunity emerged in lymphoid cells of cartilaginous fishes (i.e. sharks and rays). Adaptive immune response is characterised by enzymatic machinery specialising in the random recombination of gene segments encoding the variable parts of B-cell receptor (BCR) and T-cell receptor (TCR) for antigens. The emergence of this sophisticated defence system exerted a very potent pressure for the appearance of lymphoid structures and pathways necessary to impose immunological self-tolerance, i.e. the inability of the immune system to react against the host organism. The first thymus appeared in cartilaginous fishes concomitantly or very shortly after adaptive immunity. This organ is not an endocrine gland, but it stands at the crucial intersection between the immune and neuroendocrine systems. ${ }^{1}$ Among all lymphoid tissues, the thymus is unique in that it is the only place where there is a permanent confrontation between ancient, almost constant, neuroendocrine principles and a more recently evolved system equipped with machinery that stochastically generates diversity of the adaptive immune response. The progressive rise in the level of immune diversity and complexity may explain why failures in self-tolerance (systemic and organ-specific autoimmune diseases) are increasingly detected during evolution, with most such failures observed in the human species. Because the intrathymic education is so complex, mistakes easily occur, leading to thymic output of self-reactive T lymphocytes orientated against components of the neuroendocrine system and other peripheral tissues. Contrary to the assumption that has prevailed for a very long time, the thymus operates throughout life, and it plays a fundamental role in generating the peripheral repertoire of $T$ lymphocytes that are diverse, selftolerant and competent against non-self antigens.

\section{Thymus Physiology and the Nature of 'Neuroendocrine Self'}

The thymus constitutes the central arm of immunological self-tolerance by two essential mechanisms that are complementary and intimately associated: clonal deletion of self-reactive $T$ cells issued from the random recombination of TCR genes (negative selection), and generation of self-antigen-specific natural regulatory T cells (nTreg) that are able to inactivate in peripheral self-reactive T cells having escaped intrathymic negative selection. ${ }^{2}$

For a long time, it was thought that tissue-specific antigens targeted by autoimmune responses were sequestered from $T$ cells during their differentiation in the thymus. However, we and several other groups have demonstrated that thymic epithelial cells (TECS) from different species constitute a site for promiscuous transcription of a great number of genes belonging to neuroendocrine families, such as the neurohypophysial family, ${ }^{3}$ tachykinins, ${ }^{4}$ neurotensins ${ }^{5}$ and the insulin family. ${ }^{6,7}$ The nature of neuroendocrine self was then defined as follows: ${ }^{7}$ 
- Neuroendocrine self-antigens usually correspond to peptide sequences that have been the most conserved throughout the evolution of a given family.

- Some hierarchy characterises their expression profile in the thymus. For the neurohypophysial family, oxytocin (OT) is the dominant peptide expressed in TECs from different species. OT binding to neurohypophysial receptors expressed by thymic $T$ cells promotes a rapid phosphorylation of focal adhesion-related kinases, ${ }^{8}$ and this event could play an important role in the induction of immune synapses between TECs and developing pre-T cells. For the insulin family, insulin-like growth factor 2 (IGF-2) is the dominant peptide expressed by cortical and medullary TECS, while IGF-1 is detected in thymic macrophages, and insulin in very rare subsets of medullary TECs. ${ }^{9}$ Such a hierarchy is significant because the level of tolerance to a protein is highly correlated to the level of its expression in the thymus. High IGF-2 concentration in the thymus may explain why this protein is highly tolerated, whereas intrinsic immunogenicity of insulin may actually result due to its low expression in rare medullary TEC subsets. Furthermore, in murine foetal thymic organ cultures, the blockade of signalling between IGFs and their receptors (but not the blockade of proinsulin) severely interferes with intrathymic T-cell proliferation and differentiation. ${ }^{10}$

- In the thymus, neuroendocrine precursors are not processed according to the classic model of neurosecretion, but through pathways of antigen presentation by, or in association with, proteins of the major histocompatibility complex (MHC). ${ }^{5}$

This hierarchy in the organisation of the thymic repertoire of neuroendocrine self-antigens is also significant from an evolutionary point of view. As discussed above, some physiological and essential functions emerged before the development of adaptive immunity had to be protected from its inherent risk of autotoxicity. Therefore, OT as a peptide, implicated at different steps of reproductive biology and thus in species preservation, had to be protected to a greater degree than vasopressin (VP), which essentially controls water metabolism. Also, IGF-2 as a major growth factor in foetal development had to be protected to a greater extent than insulin, which is responsible for glucose homeostasis. Nevertheless, because of their homology, neuroendocrine self-antigens expressed in the thymus may promote cross-tolerance to other members of their respective families. This was recently demonstrated by the weaker tolerance to insulin of 1 gf 2 mice compared with wild-type mice. ${ }^{11}$ Further insight into the discrimination between the respective power of central and peripheral arms of immunological self-tolerance will be gained through the generation of mice with TEC-specific Igf2 deletion that is under current development in our laboratory.

\section{Failure in Thymus Programming of Central Self-tolerance as an Initial Event in the Development of Type 1 Diabetes and Autoimmune Endocrinopathies (see Figure 1)}

We have previously postulated that a defect in T-cell education to recognise and tolerate OT in the thymus could play a crucial role in the development of an autoimmune response selective of hypothalamic magnocellular neurones leading to an 'idiopathic' central diabetes insipidus. ${ }^{12}$ As formulated by Burnet in 1973, the essential pathogenesis of autoimmune diseases may first depend on the appearance of 'forbidden' self-reactive T cells in the peripheral T-cell repertoire. ${ }^{13}$ Since the thymus is the first site implicated in the establishment of immunological self-tolerance, thorough investigation of the mechanisms

\section{Figure 1: Thymus-dependent Programming of Central T-cell Self-tolerance and Role of Thymus Dysfunction in the Development of Type 1 Diabetes and Autoimmune Endocrine Diseases}

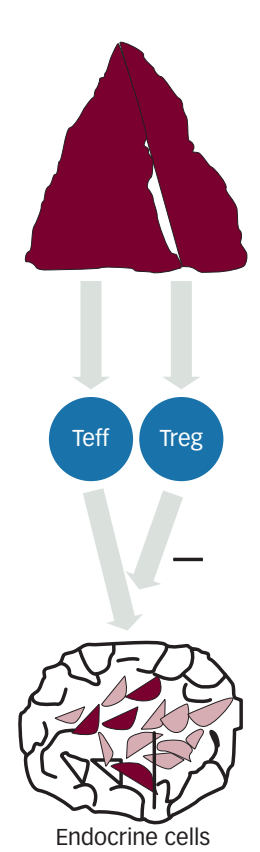

Thymus Physiology
Transcription by thymic epithelium of a series of
neuroendocrine-related genes
Deletion of T cells expressing a TCR with high affinity
for neuroendocrine self-antigens
Generation of natural Treg Foxp3+, specific for
neuroendocrine self-antigens

Control by AIRE of the intrathymic transcription of

neuroendocrine self-antigens

Thymus Pathophysiology

Absence of or decrease in thymic

expression/presentation of neuroendocrine self-

antigens (APECED/APS-I syndrome, Aire-/- mouse, NOD

mouse, $\mathrm{BB}$ rat, etc.)

Enrichment of T-cell peripheral pool with

neuroendocrine self-reactive $T$ cells

Decrease in neuroendocrine self-specific natural

Treg generation

Molecular Bridge Between Self-reactive T cells and Endocrine Targets

Influence of genetic elements intra- and extra-MHC Influence of environmental factors (virus, diet, stress)

During whole life, the thymus generates effector $T$ cells (Teff) that are competent against non-self antigens and self-tolerant, as well as self-specific natural regulatory T cells (Treg). Thymic epithelium is the site of expression for many genes encoding neuroendocrine selfantigens and tissue-restricted antigens. The AIRE gene controls transcription of most of those genes. Self-antigen expression in the thymus intervenes both in negative selection of self-reactive $T$ cells and in positive selection of self-antigen specific natural Treg. Absence of or decrease in thymic self-antigen expression as observed in several animal models of autoimmunity will lead to the enrichment of the peripheral pool with self-reactive T cells, while generation of self-antigen-specific natural Treg will be significantly decreased. Both genetic and environmental factors seem to be involved in the establishment of a molecular 'bridge' between self-reactive T cells and the endocrine antigenic targets. Once this bridge is formed, the autoimmune pathogenic process can be triggered and progressively leads to one particular endocrine deficiency.

responsible for a failure of thymic censorship will provide the scientific community with novel important keys for understanding the development of autoimmune processes. This was the essential objective of the FP6 Integrated Project Euro-Thymaide (www.eurothymaide.org) co-ordinated by the University of Liège. Abnormalities of the morphology and cytoarchitecture of the thymus have been described in several autoimmune diseases. Apoptosis of self-reactive T cells is also defective in the thymus of the NOD mouse, an animal model of human type 1 diabetes. ${ }^{14}$ Transcription of insulin-related genes (Ins, Igf1 and Igf2) has been analysed in the thymus of both diabetes-prone (BB-DP) and diabetes-resistant (BB-DR) rats, another animal model of type 1 diabetes. Ins and Igf1 transcripts were detected in all thymi from BB-DP and BBDR rats. Igf2 transcripts were also present in the thymus from all BB-DR rats, but were absent in the thymus from more than $80 \%$ of BB-DP rats, in close concordance with the incidence of autoimmune diabetes in those rats (86\%). ${ }^{15}$ This defect of Igf2 transcription in the thymus of BB-DP rats may contribute to both their lymphopenia (including absence of nTreg) and to the absence of central self-tolerance to insulin-secreting islet cells. Other authors have shown that susceptibility to autoimmune diabetes is correlated with the level of Ins2 transcription in the mouse thymus. ${ }^{16,17}$ Compared with the human foetus, with long alleles (class III) of variable number of tandem repeats (VNTR) located upstream of the INS and IGF2 loci, INS transcripts are lower in the thymus of human foetus carrying short alleles (class II) of VNTR, an important genetic trait determining susceptibility to type 1 diabetes (IDDM2). ${ }^{18,19}$ 
The demonstration that TECs are also the site for transcription of genes encoding thyroid antigens (thyroglobulin, thyroperoxydase and thyroidsecreting hormone [TSH] receptor) strongly suggests that central selftolerance to thyrocytes also concerns these proteins specific to thyroid metabolism. 20,21 Thymic hyperplasia has been described in patients with Graves' disease (type 3 autoimmune disease). This thymic hyperplasia regresses after treatment, and is not found in other cases of thyrotoxicosis from other origins (such as toxic thyroid adenoma). ${ }^{22}$

The identification of the autolmmune regulator gene (AIRE) resulted in further demonstration that thymus dysfunction plays a crucial role in the pathogenesis of organ-specific autoimmune diseases. ${ }^{23}$ AIRE single mutations are responsible for a rare autosomal recessive disorder named autoimmune polyendocrine syndrome type 1 (APS-I) or autoimmune polyendocrinopathy, candidiasis and ectodermal dystrophy (APECED, Online Mendelian Inheritance in Man [OMIM] 240300). This syndrome develops in early childhood and is characterised by multiorgan autoimmunity and hypofunction of several endocrine organs such as the parathyroid glands, adrenal cortex and sexual gonads. AIRE codes for a $54 \mathrm{kD}$ protein sharing structural characteristics with transcription factors, and AIRE expression is maximal in the thymus, mainly in medullary TECs. Depending on their genetic background, Aire ${ }^{-}$mice exhibit several signs of peripheral autoimmunity, which are associated with a very significant decrease in thymus transcription of neuroendocrine and tissue-specific genes (including Ot, Npy, Igf2 and Ins2). ${ }^{24} \mathrm{~A}$ recent study has also shown that both AIRE and VNTR class II alleles (IDDM2) determine the level of INS transcription in the human thymus. ${ }^{25}$

Although hypoparathyroidism is a hallmark of APS-1, a candidate parathyroid autoantigen has only recently been identified as the NACHT leucine-rich-repeat protein 5 (NALP5), ${ }^{26}$ which is selectively expressed in the cytoplasm of parathyroid cells. Interestingly, the NALP protein/gene family includes 14 members, and only NALP6 and NALP9 transcripts have been identified in the human thymus, suggesting that they could be responsible for inducing central self-tolerance to the whole family.

The presence of a balance between predisposing and protective loci that determines individual genetic susceptibility is not sufficient to trigger autoimmune diseases. In addition to these genetic components, environmental factors (such as viral infections, nutrition, lifestyle and even stressful events) are implicated in the pathogenesis of these disorders. With regard to type 1 diabetes, we have shown that infection of human and murine thymus with the diabetogenic coxsackievirus B4 (CVB4) results in a severe thymus dysfunction that could enhance CVB4 virulence through induction of tolerance to CVB4 and play a role in the breakdown of central self-tolerance to islet $\beta$ cells. ${ }^{27.28}$ Nevertheless, genetic factors are also implicated in the modulation of environmental triggers for autoimmune diseases, and very recently $I F I H 1$, a gene implicated in interferon response to viral RNA infections, was shown to protect against type 1 diabetes. $^{29}$

\section{Conclusions and Perspectives}

During last few years, many studies have clearly demonstrated the crucial role played by the thymus in programming central self-tolerance. More and more experimental arguments argue for a defect in thymic (central) tolerogenic function as an initial event in the development of organ-specific autoimmune diseases such as type 1 diabetes and other autoimmune endocrinopathies. According to their processing either as cognate ligands for neuroendocrine receptors expressed by immature $\mathrm{T}$ cells or as self-antigens presented by thymic MHC proteins, thymic neuroendocrine precursors exert a dual role that transposes at the molecular level the dual role played by the thymus in T-cell development and negative selection. There exists a close homology, but not an absolute identity, between these neuroendocrine self-antigens and the peripheral targets of autoimmune responses. VP is the autoantigen targeted in some cases of 'idiopathic' central diabetes insipidus, ${ }^{30-32}$ while tolerance to OT is so strong that cases of autoimmune response against OT have never been described. Analogously, insulin is the primary immunogenic autoantigen in type 1 diabetes, ${ }^{33,34}$ while tolerance to IGF-2 is very difficult to break. According to Burnet, immunological tolerance to an antigen when established during foetal life is supposed to be 'unbreakable'. If future studies confirm that the high level of tolerance to a thymic self-antigen is correlated with the active tolerogenic properties of this self-antigen in periphery, a novel type of vaccination could be developed. Contrary to classic vaccination, which is the source of immunogenic response and immune memory, negative/regulatory 'self-vaccination' could help in reprogramming immunological self-tolerance, which is absent in autoimmune diseases from foetal life and development. ${ }^{35}$

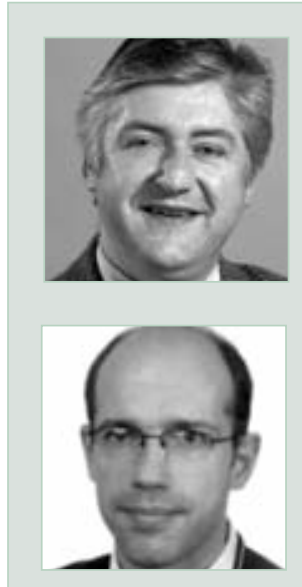

Vincent Geenen is Director of Research at the Belgian Fund of Scientific Research (FRS-FNRS), a Professor of Embryology at the University of Liège and Clinical Head of Endocrinology at the University Hospital of Liège. His research focuses on immunological tolerance to self and to embryo, the role of thymus dysfunction in autoimmunity and the development of a thymus-based negative/regulatory self-vaccine against type 1 diabetes

Olivier Dardenne is a Research Associate at the Centre of Immunoendocrinology at the University of Liège. As a post-doctoral fellow at McGill University, he engineered a mice model for pseudo-vitamin D deficiency rickets, for which he received a 2000 Young Investigator Award from the American Society of Bone and Mineral Research (ASMBR). His current research is dedicated to the engineering a transgenic mouse with Igf2 deletion targeted in thymic epithelium.
1. Geenen V, et al., J Endocrinol Invest, 1992;15:621-9.

2. Kyewski B, Klein L, Annu Rev Immunol, 2006;24:571-605

3. Geenen V, et al., Science, 1986;232:508-11.

4. Ericsson A, et al., Mol Endocr, 1990:4:1211-19.

5. Vanneste $Y$, et al., J Neuroimmunol, 1997;76:161-6.

6. Geenen V, et al., Thymus, 1993:21:115-21.

7. Martens H, et al., Immunol Today, 1996;17:312-17.

8. Martens H, et al., Neuroendocrinology, 1998;67:282-9.

9. Geenen V, Horm Metab Res, 2003:35:656-63.

10. Kecha O, et al., Endocrinology, 2000;141:1209-17.

11. Hansenne I, et al., I Immunol, 2006:176:4651-7.

12. Robert $F$, et al., Dev Immunol, 1992:2:131-40.

13. Burnet FM, Aust J Exp Biol Med Sci, 1973;50:1-9.
14. Kishimoto H, Sprent J, Nat Immunol, 2001:2:1025-31

15. Kecha-Kamoun O, et al., Diabetes Metab Res Rev, 2001;17:146-52.

16. Chentoufi AA, Polychronakos C, Diabetes, 2002;51:1383-90

17. Thebault-Beaumont K, et al., J Clin Invest, 2003,111:851-7.

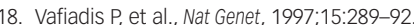

19. Pugliese A, et al., Nat Genet, 1997;15:293-7.

20. Paschke R, Geenen V, J Mol Med, 1995;73:577-80.

21. Spitzweg C, et al., Thyroid, 1999:9:133-41.

22. Murakami M, et al., J Clin Invest, 1996;98:2228-34.

23. The Finnish-German APECED Consortium. Nat Genet, 1997:17:399-403

24. Anderson MS, et al., Science, 2002;298:1395-1401.
25. Sabater $L$, et al. J Autoimmun, 2005:25:312-18.

26. Alimohammadi M, et al., N Eng/ J Med, 2008;358:1018-28.

27. Brilot $F$, et al., J Virol, 2002;76:5260-65.

28. Brilot $F$, et al., J Virol, 2004:78:9854-9861

29. Nejentsev S, et al., Science, 2009;324:387-9.

30. Scherbaum WA, et al., J Pediatr, 1985:107:922-5

31. Imura H, et al., N Engl J Med, 1993;329:683-7.

32. De Bellis A, et al., In: Geenen V, Chrousos GP (eds), Immunoendocrinology in Health and Disease, New York: Marce Dekker, Inc, 2004;439-60.

33. Kent SC, et al., Nature, 2005;435:224-8.

34. Nakayama M, et al., Nature, 2005:435:220-23.

35. Geenen V, Ann NY Acad Sci, 2006;1088:284-96. 